ESJ Social Sciences

\title{
Diseño Y Construcción De Un Prototipo Multisensorial Para El Diagnóstico Del Trastorno Por Déficit De Atención (TDA)
}

\author{
Héctor Mendoza \\ Manuel Cortez \\ Milky Rodriguez \\ Svetlana de Tristán \\ Ericka Matus \\ Jay Molino
}

Departmento de Biociencias y Salud Pública, Corregimiento de Ancón, Albrook, Universidad Especializada de las Américas

\section{Lorena Matus}

Department of Psicología, Universidad Iberoamericana, Santa Fe, Ciudad de México, México

Doi:10.19044/esj.2021.v17n3p12

Submitted: 05 November 2020

Accepted: 16 December 2020

Published: 31 January 2021
Copyright 2021 Author(s)

Under Creative Commons BY-NC-ND

4.0 OPEN ACCESS

Cite As:

Mendoza H., Cortez M., Rodriguez M., de Tristán S., Matus E., Molino J. \& Matus L. (2021). Diseño y construcción de un prototipo multisensorial para el diagnóstico del trastorno por déficit de atención (TDA). European Scientific Journal, ESJ, 17(3), 12.

https://doi.org/10.19044/esj.2021.v17n3p12

\section{Resumen}

Un prototipo para el diagnóstico del grado de déficit de atención en niños, jóvenes y adultos fue diseñado integrando diferentes pruebas sensoriales de tiempo de reacción, memoria a corto plazo, localización sonora y coordinación por colores, que son pruebas neuropsicológicas estandarizadas en la exploración del trastorno por déficit de atención e hiperactividad (TDAH). Treinta adultos saludables, en edades comprendidas entre los $20 \mathrm{y}$ 30 años, fueron reclutados de manera no aleatoria para probar el dispositivo y validar su funcionamiento. El equipo también registra los datos de cada usuario a través de un software diseñado para registrar el desempeño. El prototipo registró un tiempo de reacción promedio de $17 \pm 6.8$ segundos para la prueba de velocidad de reacción y $11.9 \pm 6.8$ segundos para la prueba de memoria. Los lenguajes de programación utilizados para el desarrollo del prototipo fueron: C++, HTML, PHP, CSS y SQL. Todos los resultados fueron 
registrados en un servidor, lo que permitiría a un especialista llevar una ficha clínica detallada del paciente.

Palabras Claves: Tiempo De Reacción, Memoria, Coordinación, Audición, Localización, Pruebas De Integración Sensorial, Déficit De Atención

\title{
Design and Construction of the Multisensory Test: An Attention Deficit Disorder Diagnostic Device
}

\author{
Héctor Mendoza \\ Manuel Cortez \\ Milky Rodriguez \\ Svetlana de Tristán \\ Ericka Matus \\ Jay Molino
}

Departmento de Biociencias y Salud Pública, Corregimiento de Ancón, Albrook, Universidad Especializada de las Américas

\section{Lorena Matus}

Department of Psicología, Universidad Iberoamericana, Santa Fe, Ciudad de México, México

Doi:10.19044/esj.2021.v17n3p1

\begin{abstract}
A prototype for the diagnosis of the degree of attention deficit in children, youth, and adults was designed by integrating different sensory tests of reaction time, short-term memory, sound localization, and color coordination, which are standardized neuropsychological tests in the exploration of the Disorder. of Attention Deficit and Hyperactivity (ADHD). Thirty healthy adults between the ages of 20 and 30 were recruited in a nonrandom way to use the prototype and validate its operation. The prototype also records the data of each user through software designed to record user performance. The prototype recorded an average reaction time of $17 \pm 6.8$ seconds for the reaction rate test and $11.9 \pm 6.8$ seconds for the memory test. The programming languages used for developing the prototype were: $\mathrm{C}++$, HTML, PHP, CSS, and SQL. All the results were recorded on a server, which would allow a specialist to keep a detailed clinical record of the patient.
\end{abstract}

Keywords: Reaction Time, Memory, Coordination, Hearing, Localization, Sensory Integration Tests, Attention Deficit. 


\section{Introducción}

El Trastorno por Déficit Atencional e Hiperactividad (TDAH) es una condición de naturaleza orgánica, con bases neurológicas que se manifiesta en la dificultad de una persona para focalizar su atención y concentración. Se exterioriza por la inquietud motora, cuyos movimientos podrían interferir en el control y en la disposición para enfrentar las actividades diarias. En Panamá el TDAH presenta prevalencia del 7.4\% de la población, siendo el $1.8 \%$ del tipo inatento, 3.2\% del tipo hiperactivo-impulsivo y un $2.3 \%$ del tipo combinado (APA, 1995; Sánchez, Velarde, \& Britton, 2011).

Los niños que padecen este trastorno están expuestos a muchas experiencias de fracaso $y$, a pesar de tener un nivel de inteligencia promedio o incluso por encima de los de su edad, su rendimiento académico puede verse afectado. Este padecimiento perturba el diario vivir de la persona: en la dificultad o la falta de capacidad para realizar tareas cotidianas, trabajar, realizar deportes o actividades recreativas de manera satisfactoria. El déficit de atención, base para el aprendizaje general, impide organizar de manera adecuada la información que adquiere una persona y, en consecuencia, ofrecer respuestas óptimas. (Arnsten, 2009; Barkley, DuPaul, \& McMurray, 1990; Bonilla Sánchez, Quintanar Rojas, \& Soloviova, 2003; de la Olvera, Ortiz, \& Pérez, 2010; Pascual-castroviejo \& Madrid, 2008; Sánchez et al., 2011)

Morales et al. describen el trastorno de integración sensorial como la incapacidad que tiene un niño para poder modular, discriminar, coordinar u organizar de manera correcta las sensaciones. Inicialmente debemos asociar un trastorno de integración sensorial a patologías generalmente no atribuibles a diagnósticos neurológicos que presentan todas o alguna de las siguientes características: problemas de conducta, dificultades de aprendizaje, y dificultades motrices y de procesamiento sensorial. De hecho, el niño puede presentar uno solo, o una combinación de varios síntomas; lo cierto es que el comportamiento característico apunta hacia una de estas dos direcciones: retraso en el aprendizaje de habilidades y destrezas motoras propias de su edad cronológica, o problemas de comportamiento de la organización personal social. En los casos más complicados, existe una combinación de ambos. (Del Moral, Pastor, \& Sanz, 2013; Gimeno Morales \& Galbe Sánchez-Ventura, 2015; Millá Romero, 2006; Palacios, 2010; Rodríguez, Areces, García, Cueli, \& González-Castro, 2018)

Además de esto, el trastorno de integración sensorial puede darse combinado con otros diagnósticos como el autismo, el trastorno por déficit de atención e hiperactividad (TDAH) o trastorno de ansiedad, pero también puede darse de manera única (Narbona García, 2001). De acuerdo con Baudry et al., sus investigaciones revelan que el TDAH y TPS son, efectivamente, dos 
diagnósticos distintos, pero que frecuentemente coexisten. En su estudio de 2410 niños previamente diagnosticados con TPS o TDAH, encontró que el $60 \%$ de dichos niños padecía en realidad ambos trastornos. Un deficiente funcionamiento de la integración de la información sensorial genera en el niño problemas de comportamiento y de aprendizaje. En un estudio realizado con niños, un grupo que presenta este déficit no refleja ser diferente a los demás niños; su inteligencia se encuentra dentro del promedio o es superior. (Beaudry Bellefeuille, 2006) Cuando el problema no es muy severo, los médicos generalmente no lo diagnostican y los padres de familia no se dan cuenta hasta que el niño ingresa al colegio y presenta dificultades para aprender a leer, escribir, respetar las normas y compartir con sus compañeros (Palacios, 2010; Rodillo, 2015).

En el salón de clases, el niño con déficit en la integración sensorial se distrae por los ruidos, luces, sonidos y estímulos de muchas personas que realizan simultáneamente diferentes acciones a su alrededor. Esto hace que su cerebro se sobreestimule y responda con un exceso de actividad. El niño hiperactivo juguetea por todo el salón, no porque quiera hacerlo, sino porque está fuera de control. Su exceso de actividad es una respuesta a sensaciones que no puede desatender, pero tampoco sabe cómo organizarlas en su cerebro; la confusión creada le hace imposible concentrarse en algo y no logra comprender lo que el profesor dice o espera de él (Palacios, 2010).

Para evaluar a los pacientes con TDAH es importante definir lo que es normal. Para ello son necesarias las entrevistas clínicas con el paciente en las que se toman en cuenta: antecedentes vinculados con la hiperactividad (p. ej., trastornos del sueño, inquietud de pequeño, bajo peso al nacer, familiares con el mismo cuadro, etc.), ajuste a los criterios clínicos diagnósticos, aspectos sociofamiliares y aspectos educativos.

El diagnóstico temprano del déficit atencional e hiperactividad permitirá a los especialistas en neuropsicología llevar acabo diagnósticos, prescribir medicamentos y/o proveer terapias para corregir y mejorar áreas tales como el aprendizaje, concentración, coordinación y la conducta.

En el presente estudio se diseña, desarrolla y prueba un prototipo electrónico capaz de realizar y registrar los resultados de pruebas neuropsicológicas basadas en integración sensorial para su potencial uso en el diagnóstico del trastorno del déficit atencional e hiperactividad en niños, jóvenes y adultos. Se realizaron pruebas enfocadas en el tiempo de reacción, localización sonora, coordinación por colores y memoria a corto plazo., entre otros. (Bulling, Ward, Gellersen, \& Tröster, 2008; Fernández, Morillo, Gilibert, Carvalho, \& Bello, 2020; Rodriguez, 2018; Rodriguez, 2011; Salvucci \& Anderson, 2001; Zhan, Zhang, Mei, \& Fong, 2016). El error en el dispositivo se calculó y es menor del $0.01 \%$ (ver Anexo 1). A partir de este estudio, es posible desarrollar otros dispositivos para el diagnóstico de TDAH 
en personas con movilidad reducida empleando captadores de señales mioléctricas (Kaneko, Yamashita, \& Iramina, 2016; Keshav, Vogt-Lowell, Vahabzadeh, \& Sahin, 2019; Rodriguez, García, \& Molino, 2020)

\section{Materiales y Metodología}

El equipo diseñado posee sensores para capturar la respuesta a los estímulos visuales y sonoros. Se utilizó una impresora 3D para construir la carcasa que aloja los sensores, luces y zumbador los cuales componen el módulo sensorial.

\section{Materiales}

Se empleó un módulo de red Ethernet Arduino (5V DC, Wiznet W5100, 10/100 Mbps), un microcontrolador ATmega2560, diodo smd 5050, resistencias $R=\bar{R} \pm 5 \% \Omega$, Potenciómetro tipo trimpot, con tres pines y potencia $P=\bar{P} \pm 10 \%$, Buzzer eléctrico con un voltaje de operación de $5 \mathrm{~V}$ DC, un sensor reflector óptico, CNY70, con una distancia operativa máxima de $5 \mathrm{~mm}$ y una estructura de aluminio para ubicar los doce módulos sensoriales. Para la medición se empleó un osciloscopio FLUKE-190-204/AM y una fuente de poder de $24 \mathrm{Vdc}$, multímetro FLUKE 179TRMS.

Se empleó un módulo de red Ethernet Arduino (5V DC, Wiznet W5100, 10/100 Mbps), un microcontrolador ATmega2560, diodo smd 5050, resistencias $R=\bar{R} \pm 5 \% \Omega$, Potenciómetro tipo trimpot, con tres pines y potencia $P=\bar{P} \pm 10 \%$, Buzzer eléctrico con un voltaje de operación de $5 \mathrm{~V}$ DC, un sensor reflector óptico, CNY70, con una distancia operativa máxima de $5 \mathrm{~mm}$ y una estructura de aluminio para ubicar los doce módulos sensoriales. Para la medición se empleó un osciloscopio FLUKE-190-204/AM y una fuente de poder de $24 \mathrm{Vdc}$, multímetro FLUKE 179TRMS.

Se empleó un módulo de red Ethernet Arduino (5V DC, Wiznet W5100, 10/100 Mbps), un microcontrolador ATmega2560, diodo smd 5050, resistencias $R=\bar{R} \pm 5 \% \Omega$, Potenciómetro tipo trimpot, con tres pines y potencia $P=\bar{P} \pm 10 \%$, Buzzer eléctrico con un voltaje de operación de $5 \mathrm{~V}$ DC, un sensor reflector óptico, CNY70, con una distancia operativa máxima de $5 \mathrm{~mm}$ y una estructura de aluminio para ubicar los doce módulos sensoriales. Para la medición se empleó un osciloscopio FLUKE-190-204/AM y una fuente de poder de 24 Vdc, multímetro FLUKE 179TRMS.

Para la fabricación de la carcasa de los módulos sensoriales, se empleó una impresora 3D iPrusa con filamento de material PLA y resolución $0.4 \mathrm{~mm}$.

Para la gestión de almacenamiento de datos se utilizó un sistema basado en My-SQL x64 bit Windows 7; para el diseño y simulación de los circuitos de control del prototipo se utilizó ISIS 7 Proteus versión 7; en cuanto a la programación del sistema de control del prototipo se empleó el Idle de 
Arduino basado en lenguaje $\mathrm{C}++$, apoyados de librerías desarrolladas para el control de elementos periféricos tales como: teclado matricial, shield Ethernet, pantalla grafica con comunicación I2C. Para el desarrollo de la página web se usó el lenguaje HTML y para la personalización de la interfaz de usuario, el lenguaje de programación web CSS. El registro de los resultados y manejo de los datos interno fue necesario la utilización del lenguaje de programación web PHP al igual que para la integración de la base de datos y la creación de tablas con resultados, promedios y gráficos por cada usuario que empleó el prototipo.

\section{Población y muestra}

El universo son estudiantes de la Universidad Especializada de las Américas. La población son todos estudiantes de la cede Facultad de Biociencias y Salud Pública de UDELAS.

La muestra forma un total de 30 estudiantes universitarios en buenas condiciones físicas y mentales de la facultad Biociencias y Salud Pública de UDELAS. Estos estudiantes fueron seleccionados de manera no aleatoria, sin distinción de sexo ni edad, escogidos con la finalidad de validar la funcionalidad y satisfacción de las pruebas emitidas por el equipo.

\section{Dispositivo}

El módulo sensorial está compuesto por los siguientes componentes: Led, buzzer y (2) opto acopladores CNY70. El CNY70 posee 4 pines: Ánodo y cátodo (led infrarrojo) y colector y emisor (Fototransistor). La configuración física del led infrarrojo y el fototransistor se encuentra ubicada en paralelo. La base del fototransistor se satura cuando un objeto se encuentra enfrente del integrado, la luz infrarroja emitida por el led se refleja, y el rayo de luz llega al fototransistor. Al saturarse la base del fototransistor se obtiene una señal en el emisor (disminución de voltaje); esta señal es enviada al operacional LM324, el mismo que se está utilizando en modo comparador. En el modo comparador, el voltaje de referencia se encuentra en el input no inversor con un voltaje de referencia de $3.90 \mathrm{~V}$ y en el input inversor llega el voltaje procedente del emisor del fototransistor, siendo este un voltaje menor que el de referencia. Cuando este evento ocurre, el amplificador comparador envía una señal de 5 voltios a los pines digitales del Arduino Mega. La figura 1 muestra un diagrama del circuito electrónico 


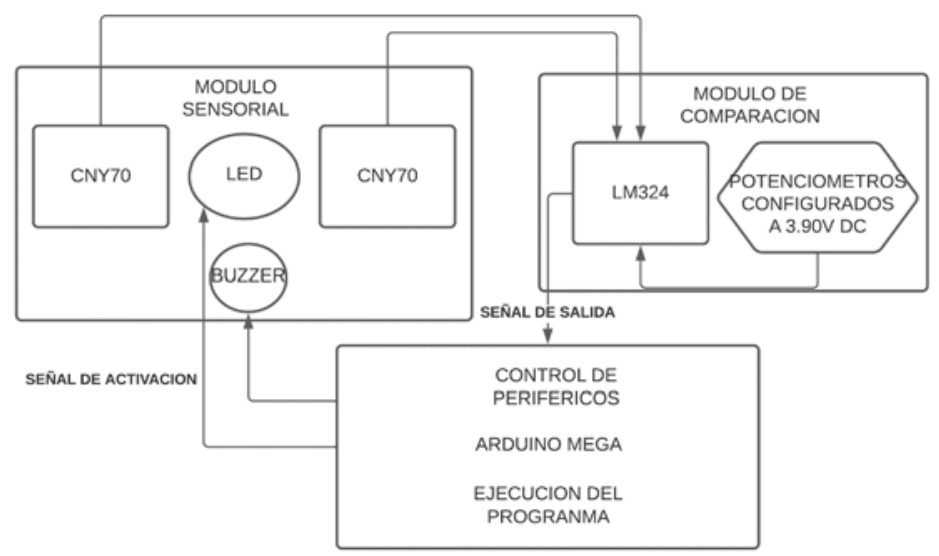

Figura 1. Flujo de señales provenientes del Microcontrolador Arduino Mega hacia el módulo sensorial para la activación del Buzzer y alimentación del sensor CNY70. Luego, la señal de activación emitida por uno de los dos sensores CNY70 es enviada hacia la etapa de comparación (LM324) y de esta se produce una señal hacia uno de los pines digitales del Arduino Mega correspondientes al módulo sensorial activado.

Cada integrado LM324 posee cuatro amplificadores en su configuración interna. Para cada módulo sensorial se están utilizando dos amplificadores, ya que cada módulo sensorial posee dos sensores ópticos CNY70, por lo que se analizan dos señales provenientes del sensor y se envía señal de respuesta a los pines digitales determinados para cada módulo respectivamente, y el mismo continúe con el siguiente bloque de programación para el test que se esté ejecutando. Durante las pruebas que realiza el prototipo, el Arduino activará aleatoriamente cada módulo sensorial. El código de programación estará esperando recibir alguna respuesta de los módulos sensoriales provenientes de los amplificadores configurados en modo comparador. Esto se muestra en la figura 2.

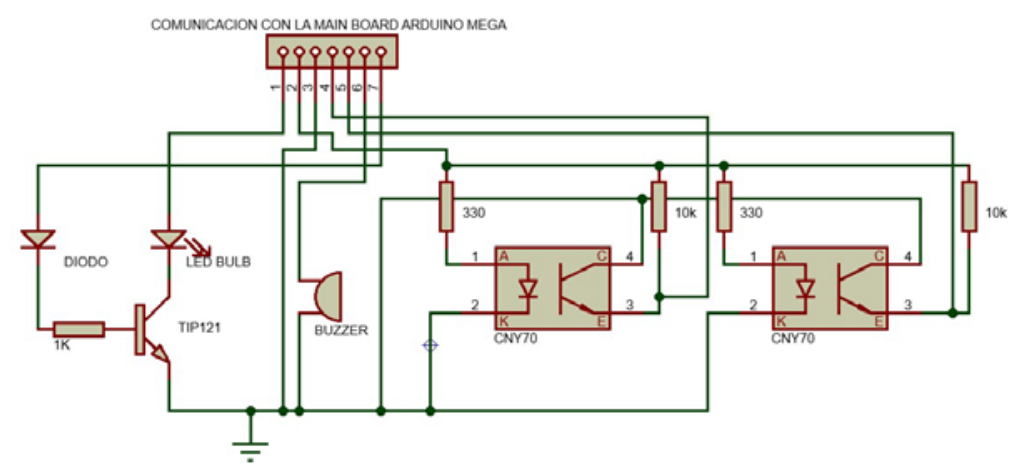

Figura 2. Módulo Sensorial: Configuración para los sensores opto eléctrico para captar la reflexión de la luz infrarroja, el buzzer para realizar el estímulo sonoro y el led bulbo activado por medio de un pulso enviado a la base del transistor NPN. 
A la terminal negativa llega la señal proveniente de los sensores opto eléctricos ubicados en los módulos sensoriales. En la salida de ambos comparadores por módulo se conectó un diodo semiconductor IN4007. Cabe destacar que, como se está utilizando dos señales provenientes de los dos optoeléctricos de cada módulo, se manejan dos señales a la par, que serían la de los dos amplificadores. Ambas salidas de los comparadores están unidas para enviar una sola señal al Arduino Mega; por lo tanto, se evita un retorno de corriente de una de la salida del amplificador al otro. Colocamos dos diodos semiconductores como se muestra en la Figura 3.

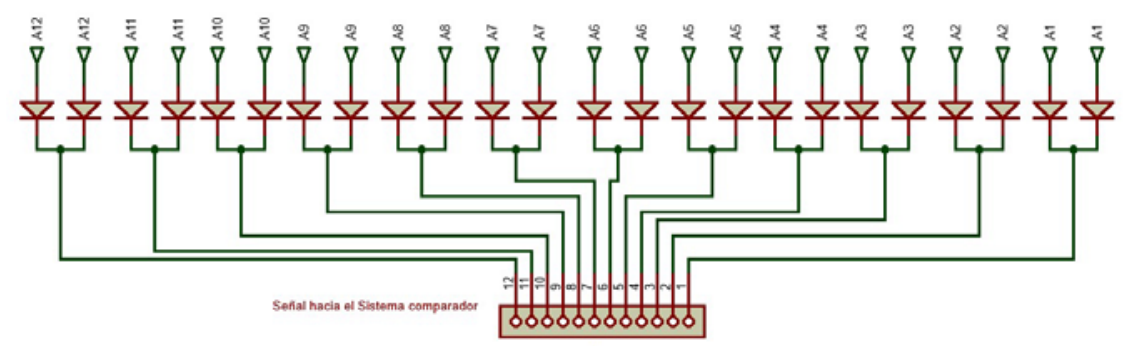

Figura 3. Diodo a cada salida para evitar el retorno de corriente al pin de salida del comparador.

El circuito comparador y los módulos sensoriales trabajan en conjunto. El LM324 usa como voltaje de operación 5v, tiene 4 amplificadores en su configuración interna, los que se configuraron como comparadores para recibir la señal de cada par de opto eléctricos de cada módulo sensorial y la señal de un potenciómetro de precisión como voltaje de referencia. El voltaje de referencia proveniente del potenciómetro de precisión o trimmer, está configurado a 3.90v. Se utilizaron 12 potenciómetros de precisión para regular el voltaje de referencia que se utilizará en el pin no inversor del LM324.

La Figura 4 muestra la carcasa fabricada con la impresora 3D, que aloja el circuito y los componentes electrónicos que conforma el módulo sensorial.

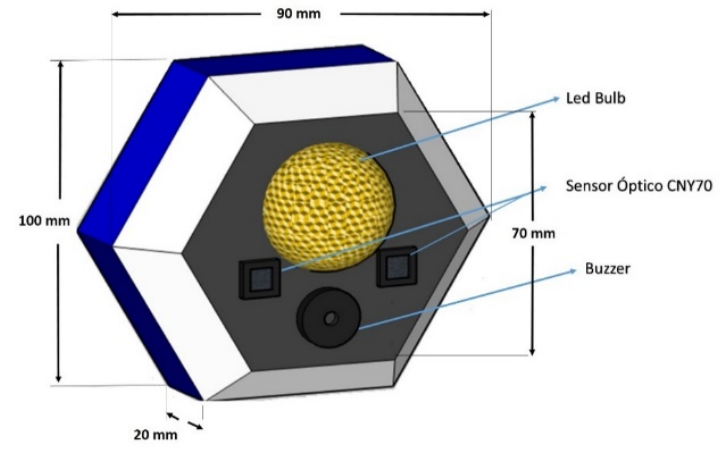

Figura 4. Carcasa que aloja los módulos sensoriales. 
En el caso del sistema para capturar datos, el gestor de base de datos MYSQL - SERVER, lo hemos programado para que ordene la información que será ingresada mediante formularios creados en nuestra página web, así como la gestión de los datos enviados por el Arduino Ethernet. Este gestor de base de datos trabajará en conjunto con nuestro emulador de servidor web llamado XAMPP.

Hemos usado el lenguaje PHP para hacer conexiones fuera de la página web como es la vinculación a la base de datos. Mediante PHP programamos que nuestra página realice una conexión, guarde y extraiga información desde una base de datos local.

Para eludir el uso de hosts o servidores externos decidimos alojar nuestro sistema de información en servidores de una red local, el cual mediante el emulador del servidor web XAMPP estará en nuestra computadora.

El Arduino Mega, una vez capte los datos obtenidos de las distintas pruebas programadas, el operador tendrá que seleccionar la opción send para realizar una comunicación de tipo serial con el Shield Ethernet. Al mismo tiempo, este módulo será el encargado de realizar la comunicación entre el Arduino Mega y la red. En el código programado en el Arduino Mega le asignamos que debe enviar los datos a una dirección específica, la cual previamente se ha configurado. Un esquema del proceso de captación de datos se muestra en el figura 5.

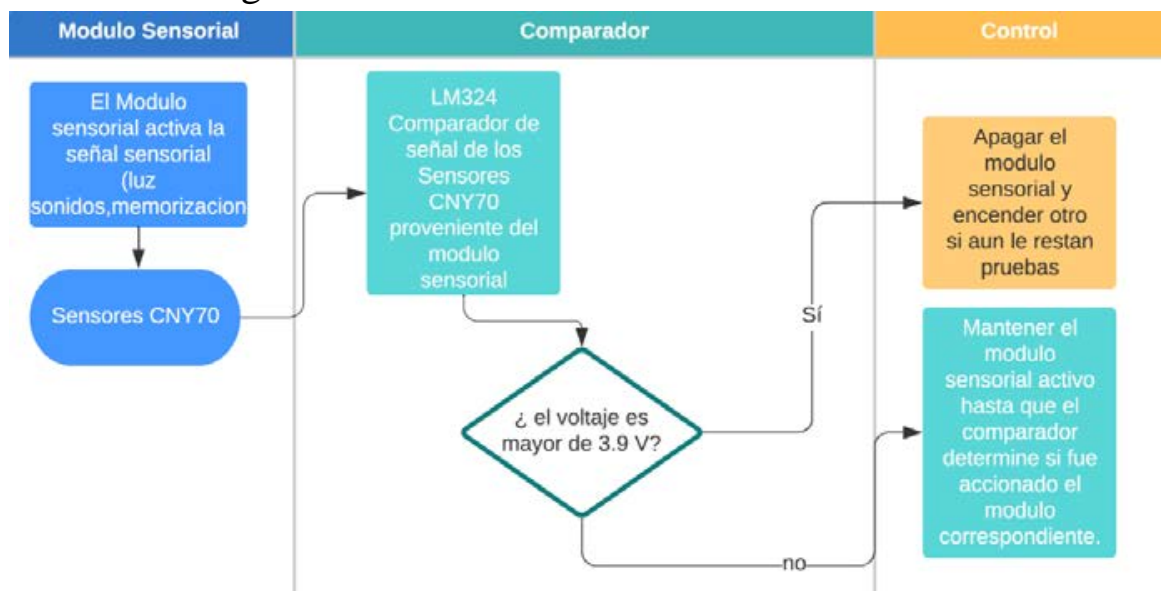

Figura 5. Elementos y Flujo del funcionamiento entre la etapa Sensorial, comparación y de control. Se muestran las dos condiciones que pueden cumplirse en la etapa de control dependiendo del estado que es percibido en la etapa de comparación por medio de la señal entrante en el integrado LM324.

\section{Pruebas}

El dispositivo Multisensorial Test permitirá aplicar cuatro tipos de pruebas que son las siguientes: Tiempo de Reacción, Memoria, Localización Sonora y Coordinación por colores. Estas se fundamentan en las pruebas de 
monitorización cognitiva computarizada (MCC 94) (Barón, Saona Olivera, \& Ramos, 2009; Etchepareborda, Paiva-Barón, \& Abad, 2009). Cada prueba fue supervisada para detectar errores y aciertos de los participantes. Se considera como un error la acción realizada por el participante que no genera un estímulo de reacción para el módulo sensorial que emite una estimulación visual o sonora y, a la vez, es capaz de censar una respuesta motriz a dicho estímulo. De igual forma, se considera un acierto cuando el participante realiza movimientos que redundan en un estímulo para el sensor. Para cada prueba existe un número fijo de secuencias que el participante debe completar. Los errores cometidos por el participante fueron tomados por el operador a cargo del equipo ya que este no cuenta con la capacidad de registrar estos eventos.

\section{Tiempo de Reacción:}

Estímulos visuales (luz) aparecen de manera aleatoria en unos módulos sensoriales. El usuario, al percibir el led encendido, debe acercar la mano a los sensores optoeléctricos que se encuentran en dicho módulo sensorial. Al activar el sensor de este módulo, el led se apagará, y un led, de otro módulo, se encenderá de forma aleatoria. Esta acción se debe realizar hasta finalizar la prueba a la cual se le mide el tiempo con el software del prototipo para determinar la reacción. Fueron veintiuna secuencias aplicadas a esta prueba de reacción sensorial aleatoria.

\section{Memoria}

La prueba consiste en leds que se encienden en un orden aleatorio, con un intervalo de un segundo por cada led. Luego de encenderse el último led de dicha secuencia, estos se apagarán y el usuario tendrá que acercar la mano a los sensores de los módulos en el mismo orden en el cual se encendieron los leds la primera vez. El tiempo en cual se ejecuta la prueba de memoria se registra por medio del software desarrollado. Fueron 7 secuencias aplicadas en esta prueba de memoria.

\section{Localización Sonora:}

Buzzers emiten tonos agudos y medios, los mismos se activan de manera aleatoria, es decir, cualquiera de los 12 módulos emitirá el sonido. El usuario debe prestar atención y tocar con su mano el módulo de dónde provenga el sonido. Luego del contacto, el buzzer del módulo se apagará y seguidamente se emitirá sonido en el siguiente módulo con una frecuencia de sonido aleatoria. Este proceso se repite hasta acabar la secuencia determinada por el software. Fueron 13 secuencias aplicadas en esta prueba. 


\section{Coordinación por Colores:}

Se asignan dos colores para cada mano; rojo para la mano izquierda y verde para la mano derecha. Estos dos colores serán los mismos que alumbren los leds del equipo. Al iniciar la prueba, se enciende el led de un módulo (de manera aleatoria). Según el color que se encienda, el usuario tendrá que tocar el módulo con la mano a la que se le asignó el mismo color. Es decir, si se enciende un módulo con el led de color rojo, el usuario tendrá que activar los sensores de dicho módulo con la mano izquierda. Al activar el sensor de este módulo, el led se apagará, y el del otro módulo se encenderá. Seguirá de esta manera hasta acabar la secuencia determinada en el sistema.

También se mide el tiempo empleado en terminar la prueba completamente y la cantidad de aciertos, los errores cometidos durante la prueba son registrados por el operador del prototipo.

\section{Conflicto de Interés}

Los investigadores reportan que no existen conflictos de intereses como se declara en el ICMJE Form for Disclosure of Potential Conflicts of Interest remitido a la dirección editorial de esta revista. Se establece que no se ha realizado ninguna prueba en pacientes con TDAH. Esto pertenece a una segunda fase del proyecto una vez se cuente con el aval de un Comité de Bioética y profesionales clínicos. Es decir, lo presentado en este documento llegó a nivel de prototipo.

\section{Resultados y Discusión}

\section{Tiempo de Reacción}

La figura 6 muestra la velocidad de reacción de cada uno de los participantes que se sometieron a la prueba. El promedio del tiempo registrado fue de $17 \pm 6.8$ segundos. Nótese que la tendencia es exponencial de decaimiento lo que indica que existe una curva de aprendizaje para manejar el dispositivo y por lo tanto aquellos participantes que esperaron más tiempo para validar el dispositivo lograron hacer el test más rápido. Esto se registra en el panel izquierdo de la figura 6 .

El panel derecho de la figura 6 muestra la cantidad de aciertos y errores que cada uno de los participantes cometió durante el proceso. 

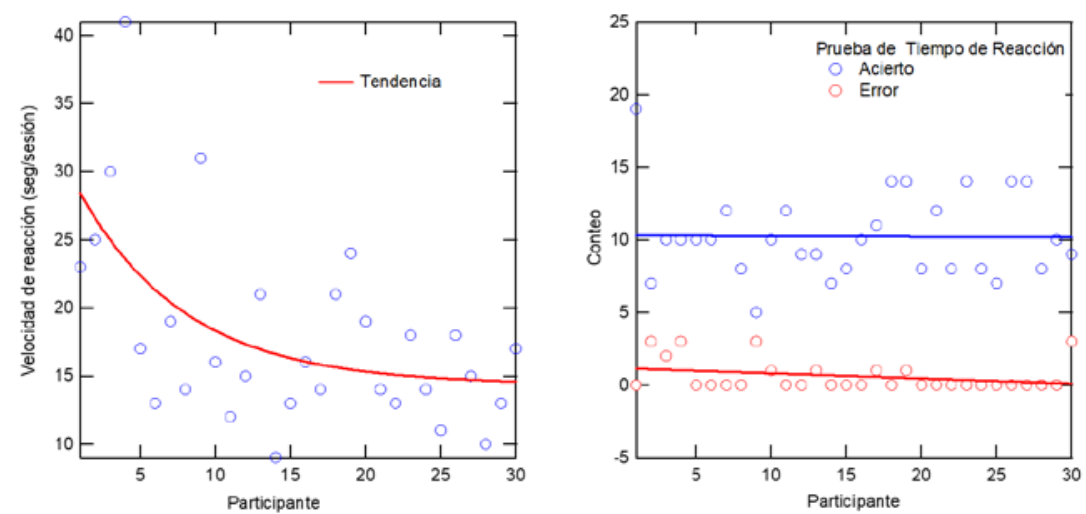

Figura 6. (Izquierda) velocidad de reacción de cada participante. (Derecha) errores y aciertos en la prueba de velocidad de reacción.

\section{Memoria}

La figura 7 muestra los resultados para el test de memoria. El tiempo promedio registrado fue de $11.9 \pm 6.8 \mathrm{seg}$. Para esta prueba la tendencia fue, también, exponencial de decaimiento lo que indica que los últimos participantes pueden realizar la tarea con mayor rapidez puesto que logran familiarizarse con el test cuando observan el desempeño de los primeros participantes. En promedio se dieron 6 aciertos y 1 error por participante. Esto se aprecia en el panel izquierdo de la figura 7. La desviación estándar para esta prueba es la misma que para la prueba de velocidad de reacción, lo que indica que pueden existir características físicas (e.g. el tamaño de las personas) lo que influyó en los resultados.
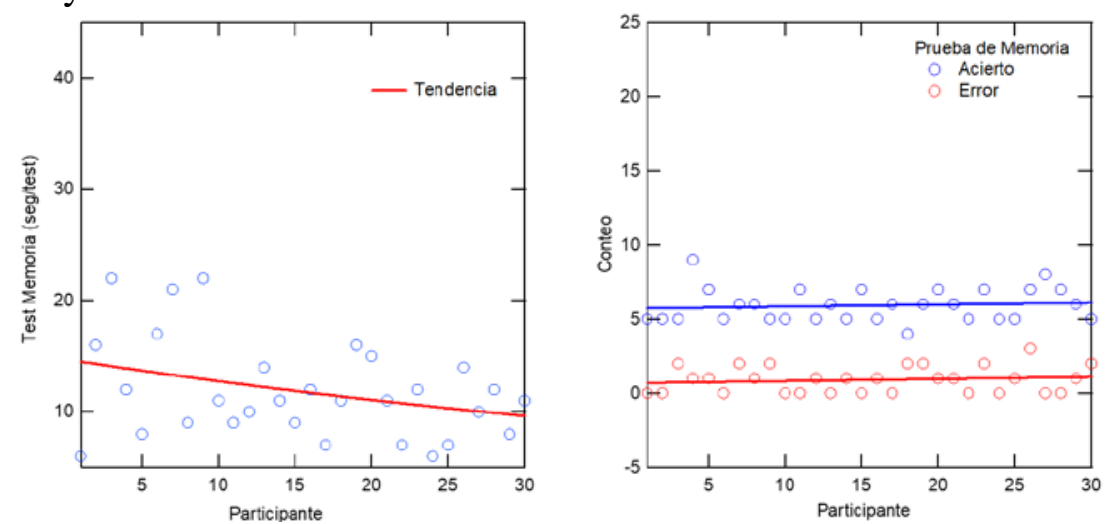

Figura 7. (Izquierda) test de memoria. (Derecha) errores y aciertos en la prueba de velocidad de reacción.

\section{Localización Sonora}

La figura 8 muestra los resultados de esta prueba. Se observa que la mayoría de los participantes realizaron la prueba en un tiempo muy prolongado, con una cantidad mínima de aciertos y una alta cantidad de errores 
cometidos. Se observa nuevamente una tendencia de decaimiento exponencial a medida en que los participantes realizaban las pruebas.
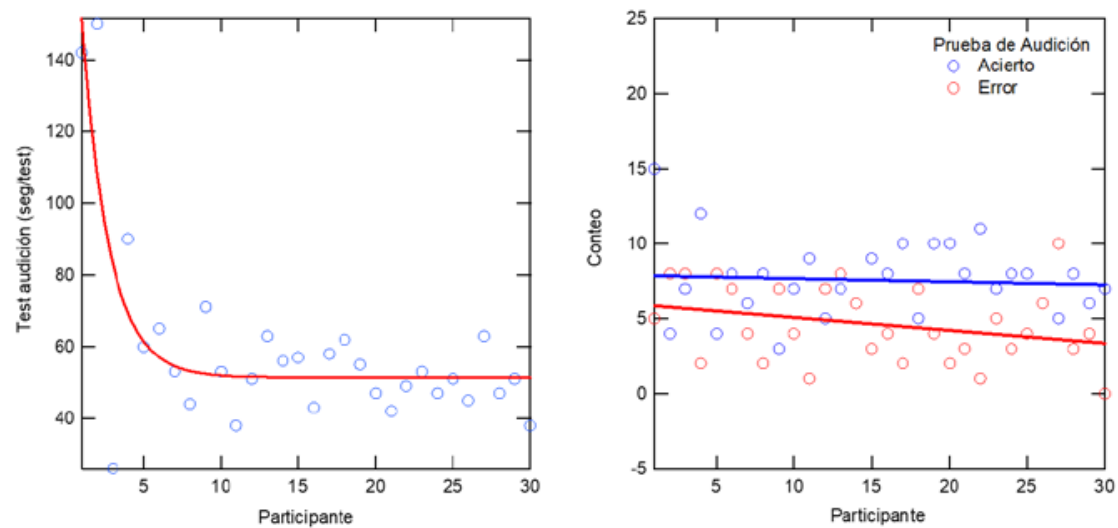

Figura 8. (izquierda) test de memoria. (derecha) errores y aciertos en la prueba de velocidad de reacción.

\section{Coordinación}

Siendo la mayoría de estudiantes los que pudieron realizar la prueba de coordinación por colores en un tiempo adecuado, con la mayor cantidad de aciertos y una mínima cantidad de errores cometidos. Se resumen los resultados en la figura 9 .
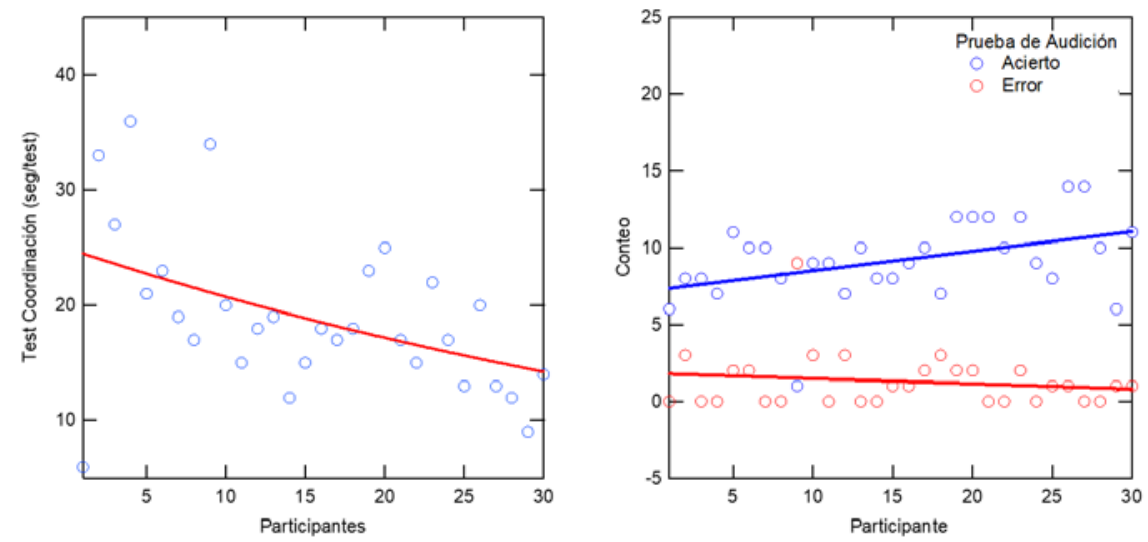

Figura 9. (Izquierda) test de memoria. (Derecha) errores y aciertos en la prueba de velocidad de reacción.

\section{Limitaciones}

El prototipo debe ser probado o evaluado, previa aprobación del protocolo en el Comité de Bioética, por un especialista en el área de Déficit de Atención y así determinar su efectividad en campo.

Dado que el prototipo es de bajo costo, es asequible para mejorar los métodos de diagnóstico del déficit atencional e hiperactividad en niños, 
jóvenes y adultos en distintas instituciones de salud en nuestro país, las cuales no tienen la facilidad de adquirir este tipo de sistemas por el elevado costo. El proyecto de investigación se limita al diseño y desarrollo de la funcionalidad del prototipo y no discute la evaluación de los resultados obtenidos por usuarios pertinentes. Estos aspectos competen a los especialistas en el área de neuropsicología o psicoterapeutas.

Existen limitaciones en el prototipo en cuanto al almacenamiento de los resultados del usuario en cada prueba, ya que de no poseer una conexión de red, el equipo no puede comunicarse con la base de datos. Esto impide que la información obtenida de las pruebas no se almacene directamente en la base de datos y se pueda visualizar en la página web. Sin embargo, el equipo permite mostrar los resultados en la pantalla, de esta manera el operario puede apuntarlos, registrarlos de forma manual y posteriormente ingresarlos manualmente al perfil del usuario en la plataforma web.

De las pruebas que ejecuta el equipo se tomarán en cuenta como resultados el tiempo total en completar la prueba, los aciertos y errores cometidos. Estas dos últimas variables no son captadas por el equipo, sino por el operario que esté supervisando al usuario al momento de realizar las pruebas. Por limitaciones en el tiempo no se pudo diseñar un mecanismo en el cual el prototipo pueda captar por sí mismo los errores cometidos.

Cabe destacar que, por trabajar con una plataforma de software abierto, se pueden realizar mejoras en el código del equipo, es decir, ajustar la precisión del conteo del tiempo, conexión con dispositivos auxiliares para la marcación de errores y aciertos del usuario al momento de realizar las pruebas y reducir más el tiempo de retraso al momento de activar los sensores optoeléctricos. Con otro microcontrolador de Arduino Mega podremos variar los tonos o frecuencias de los buzzer de acuerdo con la distancia que tenga con el usuario del equipo.

Debe implementarse el uso del Multisensorial Test, pues no es costoso, es de fácil manejo y evita los errores que puedan existir en la actualidad en el manejo de parámetros como el tiempo, los cuales deben ser muy precisos para brindar un resultado confiable.

Aunque el prototipo será utilizado para la población en general, las pruebas se realizaron en jóvenes adultos sin diagnóstico de trastorno.

Los participantes permanecieron en el mismo laboratorio al mismo tiempo, lo que les permitió aprender de los errores de los primeros. Habrá que realizar la prueba de forma individual.

De igual manera, el prototipo tiene alcances importantes en un escenario poco explorado: 1. permitirá ampliar el conocimiento de TDAH en Panamá; 2. permitiría un diagnóstico temprano; y 3. podría ser una alternativa a la medicación, particularmente de los que tienen efectos secundarios, tal como el metilfenidato que la OMS en 2019 recomendó suspender. 
Aunque ya ha habido avances sobre el uso de tecnologías para la evaluación o tratamiento del trastorno, (Fernández, Morillo, Gilibert, Carvalho, \& Bello, 2020; Kaneko, Yamashita, \& Iramina, 2016; Keshav, Vogt-Lowell, Vahabzadeh, \& Sahin, 2019) se requiere más investigación como esta de bajo costo para la población de América Latina.

\section{Financiamiento}

Esta investigación fue financiada en parte por la Universidad Especializada de las Américas a través del Fondo de Investigación No. 0052018. De igual manera agradecemos al proyecto APY-CENPEN009 financiado por la Secretaría Nacional de Ciencia y Tecnología (SENACYT) por la difusión de los resultados a través del diseño de cápsulas informativas.

\section{Conclusiones}

Por medio del uso de microcontroladores de software libre, como lo es el Arduino Mega con su lenguaje de programación C++, se diseñó y construyó un prototipo de bajo costo. Entre esas características están un menú para elección de las distintas pruebas, registro de usuario, menú de servicio técnico y conexión con base de datos para el almacenamiento de los resultados obtenidos durante las pruebas.

Se demostró la viabilidad de la plataforma diseñada para la captura y almacenamiento de datos en un servidor de la red local (se realiza una copia de seguridad). Esto permitiría tanto al especialista como al usuario (paciente) revisar sus resultados junto con una gráfica que posibilita ver el avance durante el periodo en que esté realizando sus distintas pruebas.

El diseño del equipo, liviano y armable facilita su uso portátil y sencillo para ensamblar.

El diseño se basó en teorías propuestas por especialistas en el campo de la óptica, fonoaudiología y neuropsicología, en cuanto a la configuración de los leds, posicionados de acuerdo con el campo visual de una persona; la altura y el alcance de los brazos para que realice adecuadamente el accionamiento de los sensores. La contribución en el campo del área neuropsicológica permitirá evaluar gran parte de los síntomas por desatención, hiperactividad e impulsividad de los criterios del DSM-IV, tales como: 1. Atención (No presta atención a los detalles e incurre en errores por descuido, dificultad en mantener atención en actividades lúdicas, parece no escuchar cuando se le habla directamente, no sigue instrucciones y no finaliza tareas, dificultad para realizar tareas o actividades, evita o es renuente a tareas que requieren esfuerzo mental sostenido, se distrae fácilmente por estímulos irrelevantes); 2. Hiperactividad (abandona su asiento en situaciones donde se espera que se mantenga sentado, tiene dificultad para jugar); 3. Impulsividad 
(esperar su turno, precipita respuestas antes de haber sido completadas las preguntas, interrumpe a otros).

\section{References:}

1. APA. (1995). Manual diagnóstico y estadístico de trastornos mentales. Barcelona: Masson: American Psychiatric Association.

2. Arnsten, A. F. T. (2009). ADHD and the Prefrontal Cortex. The Journal of Pediatrics, 154(5), I-S43. https://doi.org/10.1016/j.jpeds.2009.01.018

3. Barkley, R. A., DuPaul, G. J., \& McMurray, M. B. (1990). Comprehensive Evaluation of Attention Deficit Disorder With and Without Hyperactivity as Defined by Research Criteria. Journal of Consulting and Clinical Psychology, 58(6), 775-789. https://doi.org/10.1037/0022-006X.58.6.775

4. Beaudry Bellefeuille, I. (2006). Un trastorno en el procesamiento sensorial es frecuentemente la causa de problemas de aprendizaje, conducta y coordinación motriz en niños. Boletín de La Sociedad de Pediatría de Asturias, Cantabria y Castilla y León, 46(June 2006), 200_203. Retrieved from http://www.sccalp.org/bulletin_articles/418\%5Cnhttp://ibeaudry.com /BolPediatr2006_46_200203.pdf\%5Cnhttp://www.sccalp.org/documents/0000/0689/BolPediat r2006_46_197completo.pdf

5. Bonilla Sánchez, M., Quintanar Rojas, L., \& Soloviova, Y. (2003). Análisis de las funciones ejecutivas en niños con déficit de atención. Revista Española de Neuropsicología, 5(2), 163-176.

6. Bulling, A., Ward, J. A., Gellersen, H., \& Tröster, G. (2008). Robust Recognition of Reading Activity in Transit Using Wearable Electrooculography, 19-37. https://doi.org/10.1007/978-3-54079576-6_2

7. de la Olvera, F. P., Ortiz, J. D. P., \& Pérez, E. B. (2010). Cartagena’s Declaration for Attention Deficit Hyperactivity Disorder (ADHD): Breaking the Stigma. Revista Ciencias de La Salud, 8(1), 93-98.

8. Del Moral, G., Pastor, M. A., \& Sanz, P. (2013). Del Marco Teorico de Integracion Sensorial al Modelo Clinico de Intervencion. Tog, 10(May), 1-25.

9. Etchepareborda, M. C., Paiva-Barón, H., \& Abad, L. (2009). Ventajas de las baterías de exploración neuropsicoló gica en el trastorno por déficit de atención/hiperactividad. Revista de Neurologia, 48(SUPPL. 2). https://doi.org/10.33588/rn.48s02.2009073

10. Fernández, M., Morillo, M., Gilibert, N., Carvalho, C., \& Bello, S. (2020). Herramientas tecnológicas del diagnóstico y tratamiento del 
trastorno por déficit de atención e hiperactividad. The Technological Tools of the Diagnosis and Treatment of Attention Deficit Disorder and Hype Activity. (English), 80, 67. Retrieved from https://ezproxy.unav.es/login?url=https://search.ebscohost.com/login. aspx?direct=true \&AuthType=ip,url\&db=edo\&AN=142311186\&lang $=$ es $\&$ site $=$ eds-live $\&$ scope $=$ site

11. Gimeno Morales, M., \& Galbe Sánchez-Ventura, J. (2015). Trastorno por déficit de atención e hiperactividad: ¿incrementa su prevalencia o se promueve su diagnóstico? Pediatría Atención Primaria, 17(65), e95-e98. https://doi.org/10.4321/s1139-76322015000100024

12. Kaneko, M., Yamashita, Y., \& Iramina, K. (2016). Quantitative evaluation system of soft neurological signs for children with attention deficit hyperactivity disorder. Sensors (Switzerland), 16(1), 1-10. https://doi.org/10.3390/s16010116

13. Keshav, N. U., Vogt-Lowell, K., Vahabzadeh, A., \& Sahin, N. T. (2019). Digital Attention-Related Augmented-Reality Game: Significant Correlation between Student Game Performance and Validated Clinical Measures of Attention-Deficit/Hyperactivity $\begin{array}{llll}\text { Disorder } \quad \text { (ADHD). } & \text { Children, }\end{array}$ https://doi.org/10.3390/children6060072

14. Millá Romero, M. G. (2006). Atención temprana de las dificultades de aprendizaje. Revista de Neurología, 42(S02), S153. https://doi.org/10.33588/rn.42s02.2005821

15. Narbona García, J. (2001). Alta prevalencia del TDAH: ¿¿niños trastornados, o sociedad maltrecha? Revista de Neurología, 32(03), 229. https://doi.org/10.33588/rn.3203.2000632

16. Palacios, A. C. (2010). Integracion sensorial. Agapasm, 1-78.

17. Pascual-Castroviejo, I., \& Madrid, N. (2008). Trastornos por déficit de atención e hiperactividad ( TDAH ). Asociacion Española de Pediatria, 141-150. Retrieved from http://www.aeped.es/sites/default/files/documentos/20-tdah.pdf

18. Paiva Barón, H. P., Saona Olivera, G. F., \& Perna Ramos, A. P. (2009). Capacidad discriminante de las variables de la batería MCC-94 en el Trastorno por Déficit de Atención con Hiperactividad. Anales de Psicologia, 25(1), 52-59.

19. Rodillo, B. E. (2015). Trastorno por déficit de atención e hiperactividad (TDAH) en adolescentes. Revista Médica Clínica Las Condes, 26(1), 52-59. https://doi.org/10.1016/j.rmclc.2015.02.005

20. Rodríguez, C., Areces, D., García, T., Cueli, M., \& González-Castro, P. (2018). Comparison between two continuous performance tests for identifying ADHD: Traditional vs. virtual reality. International Journal of Clinical and Health Psychology, 18(3), 254-263. 
https://doi.org/10.1016/j.ijchp.2018.06.003

21. Rodriguez, M. (2011). Captación de Señales Mioeléctricas Oculares. Universidad Especializada de las Américas.

22. Rodriguez, M., García, S., \& Molino, J. (2020). Captación De Potenciales Eléctricos Oculares Para Su Uso Viable En Interfaz Hombre-Máquina, https://doi.org/10.19044/esj.2020.v16n18p102

23. Salvucci, D. D., \& Anderson, J. R. (2001). Automated eye-movement protocol analysis. Human-Computer Interaction, 16(1), 39-86. https://doi.org/10.1207/S15327051HCI1601_2

24. Sánchez, E. Y., Velarde, S., \& Britton, G. B. (2011). Estimated prevalence of attention-deficit/hyperactivity disorder in a sample of Panamanian school-aged children. Child Psychiatry and Human Development, 42(2), 243-255. https://doi.org/10.1007/s10578-0100213-2

25. Zhan, Z., Zhang, L., Mei, H., \& Fong, P. S. W. (2016). Online learners' reading ability detection based on eye-tracking sensors. Sensors (Switzerland), 16(9). https://doi.org/10.3390/s16091457 